\title{
Could a Simple Nerve Block be Considered as a Treatment Option for Occipital Neuralgia with Both Short-Term and Long-Term Effects?
}

\author{
Jeong-Woo Kwun, Young Jin Kim, Jin-Shup So \\ Department of Neurosurgery, Dankook University Hospital, Dankook University College of Medicine, Cheonan, Republic of Korea
}

Corresponding author:

\section{Young Jin Kim}

Department of Neurosurgery,

Dankook University Hospital, 201

Manghyang-ro, Dongnam-gu,

Cheonan 05278, Republic of Korea

Tel: +82-41-550-6280

Fax: +82-41-550-6284

E-mail: spine1225@naver.com

\begin{abstract}
Objective: The study aims to show both the short- and long-term treatment outcome of occipital nerve block (ONB) patients with occipital neuralgia (ON).

Methods: Patients who visited our hospital between 2013 and 2020 were reviewed retrospectively. Patients were excluded if the medical records were incomplete, if they had received a cervical operation, if they had a traumatic event prior to the symptom onset, or if the follow-up period was less than 3 months. ONB targeted the greater occipital nerve, the lesser occipital nerve or both. Short term follow-up period was defined as 3 months and long term was defined as 12 months. Injection consisted of a mixture of triamcinolone acetonide, lidocaine, and normal saline. Visual analogue scale (VAS) was recorded and compared before and during the follow-up to period to assess treatment outcome.

Results: Clinical charts of 309 candidates were reviewed and 72 patients were excluded, making it 237 patients. VAS scores significantly decreased in both 3-month follow-up (from $7.20 \pm 0.94$ to 3.48 $\pm 1.66, \mathrm{p}<0.05$ ) and after 1 year follow-up (from $7.20 \pm 0.94$ to $2.71 \pm 1.07, \mathrm{p}<0.05$ ). Only 8 patients $(3.3 \%$ ) were refractory to ONB and the procedure was found to be relatively safe since only 2 patients ( $0.8 \%)$ showed transient side effects.

Conclusion: There are many treatment options for ON. However, from the results of our study, conservative treatment via ONB may have sufficient effect in controlling symptoms of ON in both short and long term.
\end{abstract}

Key Words: Headache; Nerve block; Neuralgia
Received: September 1, 2021

Revised: September 16, 2021

Accepted: October 8, 2021 debate. The most common initial approach to this disease is pain medication, physiotherapy and a simple occipital nerve block (ONB). ONB is a straightforward method that can be carried out bluntly without the assistance of imaging tools due to the lucid anatomical landmarks of occipital nerves. Other methods such as nerve ablation, open surgery, and nerve stimulation are all viable treatment options. However, none of them seems to fully satisfy the patient for pain reduction ${ }^{20)}$.

To the author's knowledge, there is only one study regarding treatment outcome of ONBs for ON using corticosteroids and there aren't any studies that look into long-term outcomes of $\mathrm{ONB}^{10)}$. Some studies have shown that the simple nerve block method has only short-term effects (less than 2 month) making it a treatment option just for a bridging method for a more invasive measure since $\mathrm{ON}$ has a chronic aspect ${ }^{12,15,19,20)}$. However, since there is no clear answer to the cure of $\mathrm{ON}$, if symptoms can be controlled for a long and lasting time, the authors believe that more studies need to be carried out regarding non-surgical treatment methods. Therefore, the aim of this study was to find out if ONB could be a viable option for pain control for patients with $\mathrm{ON}$ in both the short- and long-term. 


\section{MATERIALS AND METHODS}

A retrospective analysis of patients, who were admitted to our hospital for $\mathrm{ON}$ and received at least one ONB from the year 2013 to 2020, was carried out. The study followed the diagnostic criteria of ICHD-3 for ON. All patients received ONB either unilateral or bilateral greater occipital nerve (GON), lesser occipital nerve $(\mathrm{LON})$, or both. The short-term results were reviewed at $3^{\text {rd }}$ month follow-up and long-term results were studied after $12^{\text {th }}$ month follow-up.

ONB was carried out at the outpatient department of our hospital, by one experienced physician who has had nerve block experience for over 12 years. It was done in a free-handed technique with no assisting imaging tools such as a C-arm, ultrasonography or computed tomography (CT).

The entry point of the injection was focused mainly on the path of the GON and LON. Injection for GON was carried out $2.0 \mathrm{~cm}$ below the external occipital protuberance and about $2.5 \mathrm{~cm}$ laterally from the midline. As for the LON the procedure was carried out $2.5 \mathrm{~cm}$ below the external occipital protuberance and about $5 \mathrm{~cm}$ laterally from the midline (Fig. 1).

Injection fluid (total volume, $8 \mathrm{cc}$ ) consisted of $4.5 \mathrm{cc}$ normal saline, 3 cc lidocaine $2 \%$, and $0.5 \mathrm{cc}$ triamcinolone acetonide $(20 \mathrm{mg} / \mathrm{cc}$ ). If patients had diabetes, the dose of triamcinolone acetonide dose was halved. Injection for each site was 2 cc. A 26-gauge spinal needle was used due to its long length which enabled our physician to insert the drug more evenly along the nerve's pathway. The drug of $1 \mathrm{cc}$ was injected first on the initial puncture site. The rest of the fluid was then inoculated in a more distal portion along the nerve's path.

Visual analogue scale (VAS) was recorded to assess treatment outcome. Its scores were documented prior to the procedure, the $2^{\text {nd }}$ week, the $3^{\text {rd }}$ month, the $6^{\text {th }}$ month, and the $12^{\text {th }}$ month after

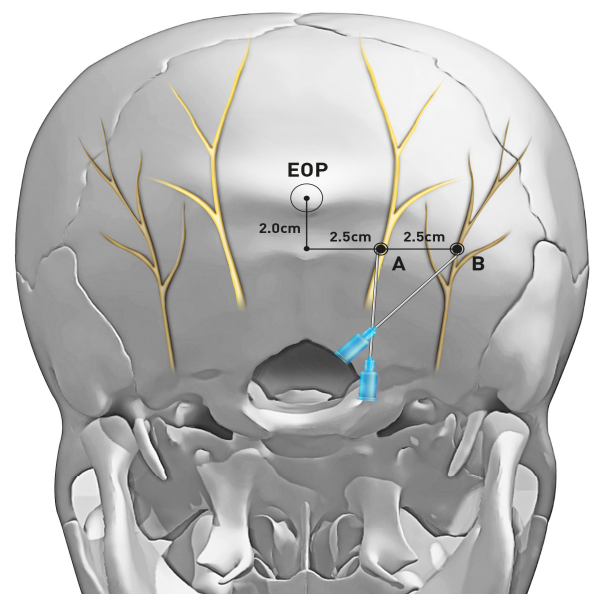

Fig. 1. The figure depicts the targets for occipital nerve block. Target $A$ is for greater occipital nerve which is $2 \mathrm{~cm}$ below and $2.5 \mathrm{~cm}$ lateral of external occipital protuberance (EOP). Target B is for lesser occipital nerve which is approximately $2.5 \mathrm{~cm}$ lateral from target $\mathrm{A}$. the procedure. Each score was compared to the initial score before ONB treatment started. If follow-up loss occurred, the VAS score of the last follow-up was recorded and compared with the initial VAS score. Some patients received more than one ONB, nevertheless, the authors compared the VAS with the initial scores. The follow- up periods in these patients were counted from the first nerve block as well, without considering the extra number of ONB.

Patients received a combination of routine medication consisting of GABAnergic drugs, non-steroidal anti-inflammatory drugs, and muscle relaxants during the follow-up period. The treatment was regarded as satisfactory when the VAS was reduced at least 50\% and the patient confirmed and stopped requesting more medication.

\section{Statistical Analysis}

The data collected were analyzed with IBM SPSS statistics version 23 (SPSS Inc., Chicago, IL, USA) for windows. Paired t-test was used to investigate if the difference in VAS between before ONB and after ONB during the follow-up period was statistically significant. The analytic results were considered significant if the p-value was less than 0.05 .

\section{RESULTS}

A total of 309 cases of $\mathrm{ON}$ receiving $\mathrm{ONB}$ were reviewed. Of the candidates, 72 patients were excluded from this study due to follow-up loss (26 patients), cervical trauma (16 patients), cervical operation (11 patients), or incomplete medical records (19 patients). Thus, the final number of candidates included in this study was 237. Their mean age was 53 (standard deviation [SD],13.6) years. There were 113 males and 124 females. The mean follow-up period was 247 (SD, 172.4) days. Co-morbidities including diabetes (9.7\%), hypertension (23.2\%), smoking (16.0\%), alcohol (25.7\%) and combined degenerative cenvical disease (45.1\%) were considered during the analysis of collected data.

The location where the ONB was carried out was GON, LON, or both. Of 237 patients, $65(27.4 \%)$ received ONB at the GON, $37(15.6 \%)$ required $\mathrm{ONB}$ at the $\mathrm{LON}$, and $135(57.0 \%)$ received ONB on both GON and LON. A total of $133(56.1 \%)$ patients received ONB unilaterally and 104 (43.9\%) received bilateral ONB. The procedure seemed fairly safe since only $2(0.8 \%)$ patients showed transient side effects such as dizziness or nausea.

The initial mean VAS score (7.20 \pm 0.94$)$ before ONB was compared with each of the mean scores during follow-up periods after ONB; $2^{\text {nd }}$ week $(2.90 \pm 1.54), 3^{\text {rd }}$ month $(3.48 \pm 1.66), 6^{\text {th }}$ month $(3.06 \pm 1.55)$, and 1 year $(2.71 \pm 1.07)$. Statistical analysis revealed that ONB was effective in decreasing pain scores to a satisfactory level compared to the initial VAS $(p<0.00001$; Table 1).

\section{DISCUSSION}

Neuralgia is defined as discomfort or pain caused by the irritation of one or more peripheral nerves ${ }^{3,20)}$. The concept of neuralgia 
along with $\mathrm{ON}$ was first introduced by Beruto and Ramos in $1821^{3,6,9}$,

12,20). $\mathrm{ON}$ is also known as Arnorld's neuralgia, C2 neuralgia, or occipital neuritis ${ }^{3,9,12)}$. In recent studies, it is standardized as $\mathrm{ON}$ and the diagnostic criterion have been introduced by $1 \mathrm{CHD}-3^{8)}$.

Symptoms regarding $\mathrm{ON}$ overlap with other types of headaches, making it difficult to diagnose for physicians. Differential diagnosis is very important since each disease entity has a different treatment approach. Physicians usually confuse $\mathrm{ON}$ with cervicogenic headache since they have many similar symptoms ${ }^{1,2,8)}$ (Table 2). One of the key differences between $\mathrm{ON}$ and cervicogenic headaches is that the range of motion of the neck for the latter is limited due to aggravation of pain caused by neck movement. In addition, the pain lasts much longer in $\mathrm{ON}$ when it occurs. Although the initial treatment can be the same for both diseases, the outcome may differ completely since cervicogenic headaches are usually due to an anatomic problem of the cervical spine ${ }^{1,2,8)}$.

$\mathrm{ON}$ may seem to be straight forward, however, it is very difficult and confusing to treat, since this disease can have numerous different causes (Table 3). Nevertheless, two main nerves involved in $\mathrm{ON}$ are GON and LON. GON is more commonly involved (90\%) than $\mathrm{LON}(10 \%)^{20)}$. Thus, understanding the anatomy of the involved nerve(s) is important for making a precise diagnosis and performing a targeted treatment plan.

Occipital nerves have three main components: GON, LON, and TON. The origin of the GON is the medial branch of the dorsal ramus of $\mathrm{C} 2$. It usually transverses the inferior oblique muscle. It may pierce through the semispinalis capitis muscle or the trapezius aponeurosis. Either way, the main function of the nerve is to provide cutaneous innervation to the posterior scalp. The LON originates from the ventral ramus of $\mathrm{C} 2$ and $\mathrm{C} 3$. The nerve usually runs parallel to the posterior aspect of sternocleidomastoid muscle and pierces

Table 1. Difference of VAS between initial and after treatment

\begin{tabular}{lcc}
\hline \hline & VAS average & Paired t-test \\
\hline Initial VAS & $7.20 \pm 0.94$ & \\
2 weeks after Tx (237 pts) & $2.90 \pm 1.54$ & \\
3 months after Tx (237 pts) & $3.48 \pm 1.66$ & $\mathrm{p}<0.00001$ \\
6 months after Tx (114 pts) & $3.06 \pm 1.55$ & \\
1 year after Tx (72 pts) & $2.71 \pm 1.07$ & \\
\hline
\end{tabular}

95\% confidence interval.

VAS: visual analogue scale; Tx: treatment; pts: patients. the deep fascia at the occiput (Fig. 2). The dorsal ramus of C3 is where TON originates. It provides innervation to the lower part of the occipital scalp and the upper cervical region. However, it is rare for the TON to be involved in $\mathrm{ON}^{3,6,12,13,15,19,20)}$

Of a total of 237 patients, most $(84.3 \%, n=200)$ patients recovered from their pain and discomfort with a single ONB. However, several patients needed more ONB to relieve their pain. The 37 patients (15.6\%) needed another ONB within two weeks. Of these 37 patients, 11 received one more nerve block (29.7\%) within 6 months of followup. Eventually, 8 patients showed refractory results to blocks after one year of follow-up. They had received 4 to 6 nerve blocks.

Results of this study revealed that ONB could be used to control discomfort and pain in most $\mathrm{ON}$ patients. However, in some patients this was not the case, even with numerous ONB and medication. This could be due to the following reasons. First, the procedure might have lacked precision since it was carried out in a free-handed technique relying only on anatomic landmarks. Second, ON might have been misdiagnosed due to its overlapping symptoms with other types of headaches and diseases. There have been case reports regarding refractory headaches to medication and nerve blocks being solved after an anterior cervical discectomy and fusion ${ }^{16)}$. Third, even though the procedure was successful, the irritated or entrapped nerve might have had irreversible injuries. In cases like this a more invasive procedure such as open surgery 5,9$)$, nerve stimulation $^{3,6,18,20)}$, nerve ablation ${ }^{3,20,21)}$ or pulsed radiofrequency method $^{3,4,6,7)}$ could be an option.

$\mathrm{ON}$ has been well described for many years. However, there is still no unanimity for its treatment methods. Management of $\mathrm{ON}$ patients can differ from patient to patient. Even with an invasive pro-

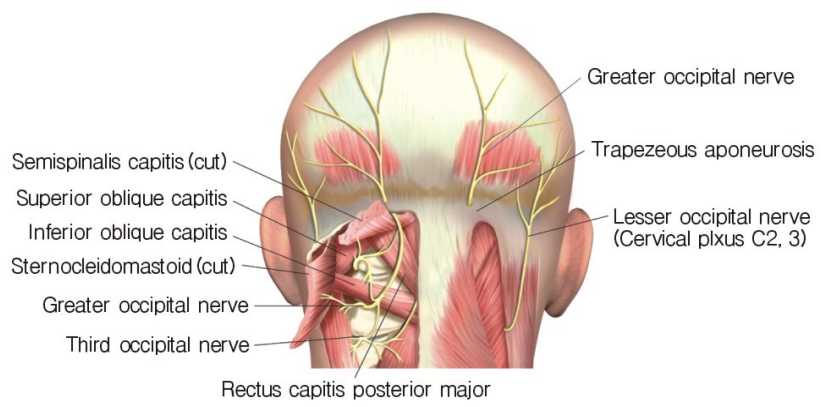

Fig. 2. Key anatomy of the occipital nerve and its surroundings showing both the muscular structures and the distribution of greater, lesser and third occipital nerves.

Table 2. Differential diagnosis of occipital neuralgia

\begin{tabular}{lll}
\hline \hline & \multicolumn{1}{c}{ Occipital neuralgia } & \multicolumn{1}{c}{ Cenvicogenic headache } \\
\hline Laterality & Unilateral, bilateral & Unilateral, no side shift \\
Severity & Moderate to severe & Moderate to severe \\
Location & Occipital area of head & Posterior neck, occipital head, fronto-parietal head, retro-orbital \\
Duration & Few sec-min & 1 hr-weeks \\
Frequency & Chronic & Chronic \\
Associated symptoms & Pain running up neck to occipital area & Similar but milder \\
Triggering factors & Muscular, vascular, neurogenic & Neck movement, pressure on neck \\
\hline
\end{tabular}


Table 3. Possible causes of occipital nerve irritation

\begin{tabular}{ll}
\hline \hline Category & Cause of nerve irritation \\
\hline Vascular & Irritation of the $\mathrm{Cl} / 2$ nerve roots by an aberrant \\
& branch of the $\mathrm{PICA}$ \\
& Dural AVF \\
& Bleeding from bulbocenvical cavernomas \\
& Giant cell arteritis \\
& Fenestrated vertebral artery pressing on $\mathrm{Cl} / \mathrm{C} 2$ nerve \\
& roots \\
& Aberrant course of the vertebral artery \\
Neurogenic & Schwannoma in the area of the craniocervical junction \\
& C2 myelitis \\
& Multiple sclerosis \\
Cl/C2 arthrosis atlantodental sclerosis & Hypermobile $\mathrm{Cl}$ posterior arch \\
& Cervical osteochondroma \\
& Osteolytic lesion of the cranium \\
& Exuberant callus formation after $\mathrm{Cl} / \mathrm{C} 2$ fracture \\
\hline
\end{tabular}

PICA: posterior inferior cerebellar artery; AVF: arteriovenous fistula. [Reprinted from "Neuralgias of the head: Occipital neuralgia", by Choi I, Jeon SR, 2016, J Korean Med Sci, 31, pp.479-88. Copyright 2016 by the Korean Academy of Medical Sciences. Reprinted with permission].

cedure, symptoms might not improve at all. From results acquired in this study, ONB not only seems to have an immediate effect, but also has a relatively lasting and satisfactory outcome in most patients. Also, the procedure itself seems relatively safe. Injection of corticosteroid and lidocaine has been found to be safe as well ${ }^{6,10,14,15)}$.

Recent studies recommend ultrasonography or CT guidance for a more accurate procedure ${ }^{11,13,17,211}$. However, considering the extra time needed for the preparation of the procedure and extra expense required if such means are used, the efficacy and simplicity of the treatment seem to be neglected. Also, considering the medical environment of an outpatient department in South Korea, a simple $\mathrm{ONB}$ is very efficient considering the time and cost. Although our study did not compare treatment results between groups who used imaging modalities and groups who did not use them, results from our study already show statistically significant and satisfactory outcomes. The goal of the nerve block is to reduce inflammatory effects by injecting a reasonable amount of steroid and lidocaine around the compromised nerve. Thus, if physicians know the general anatomic course of the affected nerve, a simple injection will usually suffice. Also, there are no anatomically critical aspects to consider around the occipital nerve, which explains why a simple procedure can have satisfactory results. Nevertheless, future studies should be carried out to prove a difference in the treatment outcomes between patients who received ONB with or without imaging modalities.

The main limitation of our study was that it was carried out retrospectively making it impossible to randomize, control or double-blind patient selection. In addition, evaluation means for treatment outcome could only be carried out with VAS, which is a rather subjective scale relying solely on the patient's response.

Also, ONB was applied simultaneously with the use of pain controlling medications. Even if patients' symptoms were relieved and sustained, this study has limitations in proving if the pain reduction was due to solely from ONB, medication or from both.

One aspect that our study failed to do was to unify the number of frequencies of ONB. During the follow-up period, numerous patients received ONB more than once if symptoms recurred even affer the first injection. The authors believed that the treatment approach of $\mathrm{ON}$ is pain management rather than cure, since $\mathrm{ON}$ has a high potential to turn chronic. Thus, the number of injections was considered not a major factor in seeing its effects in both the short or long term. Nevertheless, in order for a more accurate result, the unification of the frequencies of ONB is probably necessary.

\section{CONCLUSION}

Although there are many reasons for $\mathrm{ON}$, a simple nerve block can be administered with a relatively successful result for pain reduction. Also, since the procedure itself is relatively safe and quite easy to carry out, physicians should not be reluctant to use it as a means for controlling pain in the short and long term.

\section{CONFLICTS OF INTEREST}

No potential conflict of interest relevant to this article was reported.

\section{REFERENCES}

1. Barmherzig R, Kingston W: Occipital neuralgia and cervicogenic headache: Diagnosis and management. Curr Neurol Neurosci Rep 19:20, 2019

2. Bogduk N, Govind J: Cervicogenic headache: an assessment of the evidence on clinical diagnosis, invasive tests, and treatment. Lancet Neurol 8:959-968, 2009

3. Choi I, Jeon SR: Neuralgias of the head: Occipital neuralgia. J Korean Med Sci 31:479-488, 2016

4. Cohen SP, Peterlin BL, Fulton L, Neely ET, Kurihara C, Gupta A, et al.: Randomized, double-blind, comparative-effectiveness study comparing pulsed radiofrequency to steroid injections for occipital neuralgia or migraine with occipital nerve tenderness. Pain 156:2585-2594, 2015

5. Ducic I, Hartmann EC, Larson EE: Indications and outcomes for surgical treatment of patients with chronic migraine headaches caused by occipital neuralgia. Plast Reconstr Surg 123: 1453-1461, 2009

6. Finiels PJ, Batifol D: The treatment of occipital neuralgia: Review of 111 cases. Neurochirurgie 62:233-240, 2016

7. Huang JH, Galvagno SM, Jr., Hameed M, Wilkinson I, Erdek MA, Patel A, et al.: Occipital nerve pulsed radiofrequency treatment: a multi-center study evaluating predictors of outcome. Pain Med 13:489-497, 2012

8. International Headache Society: Headache Classification Committee of the International Headache Society (IHS) The International Classification of Headache Disorders, $3^{\text {rd }}$ edition. 
Cephalalgia 38:1-211, 2018

9. Janjua MB, Reddy S, El Ahmadieh TY, Ban VS, Ozturk AK, Hwang SW, et al.: Occipital neuralgia: A neurosurgical perspective. J Clin Neurosci 71:263-270, 2020

10. Juškys R, Šustickas G: Effectiveness of treatment of occipital neuralgia using the nerve block technique: a prospective analysis of 44 patients. Acta Med Litu 25:53-60, 2018

11. Kapoor V, Rothfus WE, Grahovac SZ, Amin Kassam SZ, Horowitz MB: Refractory occipital neuralgia: preoperative assessment with CT-guided nerve block prior to dorsal cervical rhizotomy. AJNR Am J Neuroradiol 24:2105-2110, 2003

12. López-Soto PJ, Bretones-García JM, Arroyo-García V, GarcíaRuiz M, Sánchez-Ossorio E, Rodríguez-Borrego MA: Occipital neuralgia: a noninvasive therapeutic approach. Rev Lat Am Enfermagem 26:e3067, 2018

13. Narouze S: Occipital neuralgia diagnosis and treatment: The role of ultrasound. Headache 56:801-807, 2016

14. Sahai-Srivastava S, Subhani D: Adverse effect profile of lidocaine injections for occipital nerve block in occipital neuralgia. J Headache Pain 11:519-523, 2010

15. Santos Lasaosa S, Cuadrado Pérez ML, Guerrero Peral AL, Huerta Villanueva M, Porta-Etessam J, Pozo-Rosich P, et al.: Consensus recommendations for anaesthetic peripheral nerve block. Neurologia 32:316-330, 2017
16. Schofferman J, Garges K, Goldthwaite N, Koestler M, Libby E: Upper cervical anterior diskectomy and fusion improves discogenic cervical headaches. Spine (Phila Pa 1976) 27:22402244, 2002

17. Shim JH, Ko SY, Bang MR, Jeon WJ, Cho SY, Yeom JH, et al.: Ultrasound-guided greater occipital nerve block for patients with occipital headache and short term follow up. Korean J Anesthesiol 61:50-54, 2011

18. Sweet JA, Mitchell LS, Narouze S, Sharan AD, Falowski SM, Schwalb JM, et al.: Occipital nerve stimulation for the treatment of patients with medically refractory occipital neuralgia: Congress of neurological surgeons systematic review and evidence-based guideline. Neurosurgery 77:332-341, 2015

19. Thomas DC, Patil AG, Sood R, Katzmann G: Occipital neuralgia and its management: An overview. Neurol India 69:S213S218, 2021

20. Urits I, Schwartz RH, Patel P, Zeien J, Connor D, Hasoon $\mathrm{J}$, et al.: A review of the recent findings in minimally invasive treatment options for the management of occipital neuralgia. Neurol Ther 9:229-241, 2020

21. Vanderhoek MD, Hoang HT, Goff B: Ultrasound-guided greater occipital nerve blocks and pulsed radiofrequency ablation for diagnosis and treatment of occipital neuralgia. Anesth Pain Med 3:256-259, 2013 